\section{Transient Amaurosis after Mandibular Nerve Block}

\section{Brit. med. F., 1967, 1, 681}

Though both transient and permanent amaurosis have been reported after local anaesthesia for dental extraction, the number of cases is small and the condition not widely recognized.

Walsh (1957a) noted the occurrence of ipsilateral transient amaurosis after the extraction of upper teeth, and this he attributed to the local anaesthetic reaching the retina via the rich anastomosis between the internal and external carotid arteries shown by Walsh and King (1942). Cases in which similar symptoms occurred after mandibular anaesthesia were reported by Morelli (1952), but here two cases examined ophthalmoscopically showed the typical picture of central retinal artery occlusion, apparently caused by temporary reflex angiospasm ; while a case of transient amaurosis accompanied by ocular paresis and exophthalmos was thought by Pázmányi (1955) to be due to reflex venous spasm caused by irritation of the veins at the time of the injection.

In one case each of bilateral (Walsh, 1957b, p. 1202) and unilateral (Walsh, 1957b, p. 866) permanent amaurosis, reported by Walsh, fat embolism, possibly due to intra-arterial injection of oily procaine hydrochloride, was suspected. Sokolić (1960) described a similar case where fat embolism affected the contralateral eye one day after the extraction of a lower molar tooth.

The present communication reports two cases of transient amaurosis and one of isolated external ocular muscle paresis. Intra-arterial injection of local anaesthetic in subjects with uncommon vascular patterns is believed to be the cause.

\section{Case Reports}

Case 1.-A 16-year-old girl was given a right mandibular nerve block of approximately $2 \mathrm{ml}$. of an aqueous solution of procaine and 1:300,000 adrenaline; the dental surgeon reported that the injection was given routinely, except that the latter part was injected more rapidly than usual. Immediately after completing the injection, however, he noticed two areas of transient blanching of the skin, one lateral to the eye and the other above the eyebrow on the same side as the injection. At the same time the patient became aware of numbness over the whole of the side of her face, and soon realized that the sight in that eye had gone completely; the eye became markedly divergent and adduction was impossible. After 20 minutes the sight returned and diplopia was experienced for about 10 minutes. On subsequent examination no ocular abnormality was detected.

Case 2.-A 39-year-old consultant anaesthetist was given a left mandibular nerve block before the extraction of a tooth. The injection was similar to that given in Case 1, except that the initial injection of $2 \mathrm{ml}$. was followed by a repeat injection of $1 \mathrm{ml}$. about a minute later. After this latter injection the patient noticed anaesthesia over the maxilla, as well as of the lower jaw, but nothing else untoward at the time. The tooth was painlessly extracted and he drove away approximately half an hour after the injection. Almost immediately, however, he collided with another car, which he had failed to see approaching his left-hand side. After the accident he noticed for the first time that vision in his left eye was very blurred, and on account of this consulted his general practitioner. He was examined approximately four hours after the injection, when the pupil on the affected side was found to be dilated, with a diminished light reflex; the vision and pupil soon returned to normal, and later his eyes and sight were found to be normal.

Case 3.-An ophthalmologist aged 30 experienced diplopia on looking to the right about five minutes after a right mandibular nerve block; there was no diplopia in other directions of gaze or any other unusual symptoms. The diplopia cleared after 20 minutes. The dentist, who was medically qualified, confirmed that the patient had had a transient lateral rectus palsy.

\section{Discussion}

It is unlikely that fat embolism occurred in these cases, since an oily solution of procaine was not used, and the symptoms were transient and of immediate onset. There remains, however, the possibility either of reflex angiospasm or of intra-arterial injection of local anaesthetic. Of these the latter seem to be the more likely explanation, since, though angiospasm could have accounted for the blanching of the skin in Case 1 and for the amaurosis in Cases 1 and 2, it is difficult to explain the wider field of anaesthesia experienced by these patients and the apparentiy isolated lateral rectus palsy in Case 3. Accidental intra-arterial injection of local anaesthetic might, however, explain all the signs and symptoms.

The site of injection for mandibular nerve block is immediately proximal to the point of entry of the inferior dental nerve and artery into the narrow inferior dental canal, and rapid intra-arterial injection at this point might cause back flow of the fluid along the short length of vessel between it and the parent maxillary artery. Blood from this source is unlikely to reach the retina in a patient with a normal vascular pattern' since reversal of the blood flow in the ophthalmic artery would be necessary. This is known to occur after isolation of the origin of the ophthalmic artery by ligation of the internal carotid artery above and below an aneurysm (Dandy, 1938), but it is improbable that it occurs normally. If it is assumed that the internal carotid artery was patent in the cases reported in this paper, a more feasible explanation is offered by those uncommon cases where the orbit is supplied either wholly or in part by the middle meningeal branch of the maxillary artery.

This anomaly has been well described. Singh and Dass (1960) found that the trunk of the ophthalmic artery was not patent in two of 106 orbits examined, and that in these cases the middle meningeal artery supplied the eye and orbit through the superior orbital fissure, via an anastomosis between an enlarged recurrent meningeal branch of the lacrimal division of the ophthalmic artery and the middle meningeal artery. This source augmented the orbital blood supply in a further four cases in which the ophthalmic artery was patent but much smaller than usual; the proportions of the orbit supplied from these two sources varied considerably.

It is possible that the cases reported here represent different degrees of this vascular anomaly. In Case 1 the widespread anaesthesia, the blanching of the skin, and the amaurosis with external ophthalmoplegia suggest that the orbit was entirely supplied by the middle meningeal artery, and that it was perfused by local anaesthetic and adrenaline. In Case 2 anaesthesia over the maxilla and the loss of sight might indicate substantial orbital supply from this vessel, while Case 3 suggests that blood from the middle meningeal artery entered only the lacrimal division of the ophthalmic artery, thus affecting only the area of the lateral rectus muscle.

Because of the possibility of a more serious road-traffic accident than the one in which Case 2 was involved, we feel that the occasional occurrence of temporary amaurosis after inferior dental nerve block should be more widely recognized.

The Manchester Royal Eye Hospital

P. L. BLAXTER, F.R.C.S

M. J. A. BRITTEN,* F.R.c.s.

* Present address: Stockport Infirmary, Cheshire.

\section{REFERENCES}

Dandy, W. E. (1938). Ann. Surg., 107, 654

Morelli, G. (1952). Öst. Z. Stomat., 49, 559.

Pázmányi, Gy. (1955). Acta med. hung., 8, 133.

Pazmanyi, Gy. (1955). Acta med. hung., 8, 133.

Singh, S., and Dass, R. (1960). Brit. f. Ophthal.,

Walsh, F. B. (1957a). Clinical Neuroophthalmology, 2nd ed., p. 1202. Walsh, F. B. Baltumore __ and King, A. B. (1942). Arch. Ophthal., 27, 1. 